\title{
Competition Among Manufacturers in Technological Innovation in the Market with Delayed Information
}

\author{
Jair S. Dos Santos ${ }^{1, *}$, Katia A. G. Azevedo ${ }^{1}$, Paola Torresan ${ }^{2}$ \\ ${ }^{1}$ Dep. de Computação e Matemática- FFCLRP-Universidade de São Paulo, Brazil \\ ${ }^{2}$ Faculdade de Economia e Administração de Ribeirão Preto Universidade de São Paulo, Brazil
}

\begin{abstract}
The search for information in memory of technological competition process in the market is analysed using techniques widespread in biomathematics. Here we examined the effects that delayed information causes in choosing strategies process on the part of manufacturers to supplant a technology by introducing an alternative one. A differential-equation system with delays is presented to describe the dynamics of an endogenous model with memory. Situations of competition in which the market has several manufacturers using the same technologies and are in competition are analyzed. Conditions for stability and existence of periodic oscilations by means of Hopf bifurcation is investigated.
\end{abstract}

2010 Mathematics Subject Classifications: 34K15, 34C23, 92A15, 90A60, 90A16, $91 \mathrm{~B} 55$

Key Words and Phrases: delay-differential equation, endogenous competition, stability, Hopf-bifurcations, innovation technology, delayed information.

\section{Introduction}

The sharing of the benefits generated by technological knowledge involves a variety of flows which govern actions of manufacturers and consumers of technological innovation. A number of business-cycle models postulating that both, internal factors in market as demand instability, resource avaliability or external ones, as instability of government policies, setbacks in environmental regulation, influence strongly the global dynamics of all producing activities (see $[1,2,9-11,13])$. Recently, technology planners and market agents have been interested in models that focus their attention on two causal variables, the one that captures the measure of the potential quantity of consumers in an economic system susceptible to adopt the new technology and another that captures the measure of quantity of a product with new technology (see $[1,2,7,10]$ ).

In [10], the authors have adopted the transposition of techniques used in biomathematics to analyze the changes in migration of consumers facing new technologies (see also [3]). In

*Corresponding author.

Email addresses: jair@ffclrp.usp.br (J. dos Santos), pa.torresan@gmail.com (P. Torresan) 
their model they considered external and internal socioeconomic factors but, they have assume that each firm takes only into account instantaneous information about both, its own technologic output and the one of their competitors. They show clear-cut results that describe the dynamics of the competition among manufacturers producing innovation technology. Huang, in [7], investigates what is the impact that accurate information has on the features on the dynamics of an oligopolistic market and shows how an improvement in information accuracy (or elimination of delayed information) may destabilize an existing stable equilibrium.

Our purpose is contributing to understand the basic mechanisms involved in the complexity of competition and co-existence of new technologies in innovation technology process. We will see later which techniques from dynamical systems, the memory of the production process can contain to explanation the fluctuations in indicators of innovation process technology and ensure reliable assessment of possible impacts upon the socioeconomic factors that affect both demand and supply through innovation (see $[2,14]$ ).

Form the view point of many researchers, the production process of the market depends endogenously on its past history and in several researches, on mathematical modelling taking into account this problem (see $[2,5-7,9,11,13]$ ).

The authors in [5] construct two linear continuous-time dynamic oligopoly models with partial adjustment towards the best response and analyse the effects on local stability caused by lagged information. The authors, in [6], establish a class of economic models with two delays, one of them in production process and another in consumption. They discuss the stability of the equilibrium point for economic system and the existence of Hopf bifurcation. If the economy is in recession the government uses fiscal mechanisms or monetary policy and increases its expenditure to stimulate consumption and investment (pro-cyclical policy, see [12]). Takeuchi and Yamamura, in [11], investigate how the fiscal policy with a time delay affects stability in an economy. They assume that there exists a time delay between policy making to adjust the economy and its implementation which is divided into two factors: recognizing and decision making, which are lagged actions.

Having to control the uncertainty effect about adoption of new technology, individual manufacturer attempt to delay decision making until they receive more accurate information to minimize uncertainty and to ensure improvement in performance. So, in their decisions technology planners and market agents are always concerned about the existence of time delay between the moment when it is necessary to act and the moment of recognizing the necessity of action. We assume that they act consciously and that there are lags between the time that information are obtained and the time when decisions related to it are implemented.

We propose a similar model of endogenous competition as presented by the authors, in [10]. Let $x(t)$ be the potential quantity of consumers within an economic system at instant $t$. Let us denote by $y(t)$ the quantity of a product with new technology put on the market at instant $t$. We assume that the evolution rate of the quantity of consumers in an economic system susceptible to adopt the new technology increases with the function $g$ and decreases with the sum $p_{0}$ and $p_{1}$. The function $p_{0}$ represents the functional response rate of manufacturers with time delay incorporated. The function $p_{1}$ represents the instantaneous functional response rate of manufacturers. The function $s+q(y)$ expresses the rate of specific extinction of the manufacturers due to intraspecific competition among them. The growth rate of the 
product with new technology depends on past history with $p_{0}$ and on the current moment at $p_{1}$.

$$
\begin{aligned}
& \dot{x}(t)=x(t) g(x(t))-y^{m}(t)\left[p_{0}\left(x(t-\sigma)+p_{1}(x(t))\right]\right. \\
& \dot{y}(t)=y(t)\left\{-s-q(y)+\gamma\left[p_{0}(x(t-r))+p_{1}(x(t))\right] y^{m-1}(t)\right\} .
\end{aligned}
$$

where $g, p_{0}, p_{1}, q \in C^{1}([0, \infty), R), \sigma>0, r>0$ and $m \geq 1$ is the constant of mutual interference.

Assumption 1. Let be $p(u)=p_{0}(u)+p_{1}(u)$. Assume that $p(0)=0, q(0)=0$, and $p^{\prime}(u)>0$, $q^{\prime}(u)>0$, for $u \geq 0$. Moreover, there is $u_{0}>0$ such that $g\left(u_{0}\right)=0, g(0) u>0$, $\lim _{u \rightarrow 0}\left(u g^{\prime}(u)+g(u)\right)>0, g^{\prime}(u)<0$ for $u \in[0 ; \infty)$.

The same way [10], it follows from Assumption 1 that there are $x_{0}>0$ and $y_{0}>0$ so that $P_{0}=\left(x_{0}, y_{0}\right)$ is the unique positive equilibrium point of (1), where $y_{0}=\left[\frac{x_{0} g\left(x_{0}\right)}{p\left(x_{0}\right)}\right]^{\frac{1}{m}}$ and $p$ is defined in Assumption 1.

Assumption 2. Let $\left(x_{0}, y_{0}\right)$ be the positive equilibrium point of the system (1). Le us assume that

a) $x_{0} g\left(x_{0}\right)=y_{0}^{m} p\left(x_{0}\right)=y_{0}\left(s+q\left(y_{0}\right)\right)=[(m-1) \gamma]^{-1} y_{0}^{2} q^{\prime}\left(y_{0}\right)$,

b) $p_{0}\left(x_{0}\right)=p_{1}\left(x_{0}\right), \tau_{m}=\frac{m}{m-1} y_{0} q^{\prime}\left(y_{0}\right)<1$.

c) $0<-p_{0}^{\prime}\left(x_{0}\right)<p_{1}^{\prime}\left(x_{0}\right)$ and $x_{0} g^{\prime}\left(x_{0}\right)+g\left(x_{0}\right)-y_{0}^{m} p_{1}^{\prime}\left(x_{0}\right)<0$.

System (1) becomes the system (26) in [10] if, $0<m \leq 1$ and $\sigma=r=0$. Stability of competition among new technologies available close to $P_{0}$ was analysed in [10]. Since the model (1) depends on two delays, that leads to great complexity in the analysis of competition among new technologies available on the market (see [4]). We observe that in this system the delays can not be eliminated by any change of variable. Its dynamics are studied in terms of the local stability of $P_{0}$ and of the Hopf bifurcation that is proven to exist as one of the delay crosses some critical value. To achieve our goals, we shall analyse how the roots of (3)) are distributed with respect to the imaginary axis. This is a classical problem that, in addition to being important by itself, plays an important role in the study of asymptotic behavior in the theory of delay differential equations (see [4]).

If we set $\kappa_{11}=x_{0} g^{\prime}\left(x_{0}\right)+g\left(x_{0}\right)-y_{0}^{m} p_{1}^{\prime}\left(x_{0}\right), \kappa_{12}=-m y_{0}^{m-1} p\left(x_{0}\right), \kappa_{21}=\gamma p_{1}^{\prime}\left(x_{0}\right) y_{0}^{m}$, $\kappa_{22}=(m-1) \gamma y_{0}^{m-1} p\left(x_{0}\right)-y_{0} q^{\prime}\left(y_{0}\right), b_{11}=-y_{0}^{m} p_{0}^{\prime}\left(x_{0}\right), b_{21}=\gamma y_{0}^{m} p_{0}^{\prime}\left(x_{0}\right)$, we obtain the system

$$
\left\{\begin{array}{l}
\dot{x}(t)=\kappa_{11} x(t)+\kappa_{12} y(t)+b_{11} x(t-\sigma) \\
\dot{y}(t)=\kappa_{21} x(t)+\kappa_{22} y(t)+b_{21} x(t-r)
\end{array}\right.
$$

that is the linearized system of (1), close to $P_{0}$. From Assumption $2 a$ it follows that $\kappa_{22}=0$. If $a=y_{0}^{m+1} q^{\prime}\left(y_{0}\right)\left(-p_{0}^{\prime}\left(x_{0}\right)\right), b=y_{0}^{m}\left(-p_{0}^{\prime}\left(x_{0}\right)\right), c=\gamma y_{0}^{(m+1)} q^{\prime}\left(y_{0}\right) p_{1}^{\prime}\left(x_{0}\right)$ and $d=x_{0} g^{\prime}\left(x_{0}\right)+g\left(x_{0}\right)-y_{0}^{m} p_{1}^{\prime}\left(x_{0}\right)$, then the characteristic equation of the system (2) is given by

$$
H(\lambda)=\lambda^{2}-d \lambda-b \lambda e^{-\lambda \sigma}-a e^{-\lambda r}+c=0 .
$$


Let $\lambda=x+i y$ be a solution of equation (3). Separating real and imaginary parts in (3) we obtain the following equations system for $x$ and $y$

$$
\left\{\begin{array}{l}
x^{2}-\left(y^{2}-c\right)-d x-b e^{-\sigma x}[x \cos y \sigma+y \sin y \sigma]-a e^{-r x} \cos y r=0 \\
2 x y-d y+b e^{-\sigma x}[x \sin y \sigma-y \cos y \sigma]+a e^{-r x} \sin y r=0 .
\end{array}\right.
$$

The solutions of the system (4) with null real part are solutions of the system

$$
\left\{\begin{array}{l}
b y \sin y \sigma+a \cos y r=-y^{2}+c \\
-b y \cos y \sigma+a \sin y r=d y
\end{array}\right.
$$

Suppose $y \neq 0$ is a solution of (5), then we must have

$$
\sin (\sigma-r) y=\frac{\left(-y^{2}+c\right)^{2}+d^{2} y^{2}-b^{2} y^{2}-a^{2}}{2 a b y}:=\varrho(y) .
$$

If we define $u(y)=s+q(y)$, we indicate elasticity of $u$ with respect to $y$ at $y_{0}$ by $\epsilon_{q}$. Analogously, we have $\epsilon_{g}, \epsilon_{p_{0}}, \epsilon_{p_{1}}$ and $\epsilon_{p}$ are the elasticities of $g, p_{0}, p_{1}$ and $p$ at $x_{0}$, respectively (see Assumption 1).

Remark 1. Authors in [10] consider the system (1) with $\sigma=r=0,0<m \leq 1$ and the corresponding to configuration of the equilibrium $P_{0}$ is E3 (see [10, pp. 364]). They indicate the diagonal elements of the variational matrix associated to E3 by $H=g\left(x_{0}\right)\left[\epsilon_{g}+1-\epsilon_{p}\right]$ and $R=\gamma(m-1)\left(s+q\left(y_{0}\right)\right)\left[1-\epsilon_{q}\right] \leq 0$. Using $H+R$ and $L=H R-m \gamma\left(y_{0}\right)^{2 m-1} p\left(x_{0}\right) p^{\prime}\left(x_{0}\right)$ they describe the stability of E3.

We follow the alternative offered Bléair and Mackey, [2] which make it possible an endogenous explanation for erratic behaviour of competition with memory among new technologies available, when the innovation process operates around equilibrium point. The dynamics is described in terms of elasticities (see also [8]). We also show that the dynamics of the model (1) depends, essentially, on delays and on elasticities of the rate of evolution of the consumers, on the functional response rate of manufacturers and on the rate of specific extinction of manufacturers owing to the intraspecific competition among then manufacturers.

From Assumption 2 it follows that

$$
\begin{aligned}
& b>0, c>a>0, d<0, \epsilon_{g}<0, \epsilon_{p_{1}}>2\left(1+\epsilon_{g}\right), \\
& 0<-\epsilon_{p_{0}}<\epsilon_{p_{1}}, \epsilon_{p}=\frac{\epsilon_{p_{0}}+\epsilon_{p_{1}}}{2}, \epsilon_{q}=m-1,
\end{aligned}
$$

It also follows from Assumption 2 that,

$$
\begin{aligned}
& 2 a=-\epsilon_{p_{0}} \tau_{m} g\left(x_{0}\right), 2 b=-\epsilon_{p_{0}} g\left(x_{0}\right), 2 c=\epsilon_{p_{1}} \tau_{m} g\left(x_{0}\right), \\
& 2 d=g\left(x_{0}\right)\left[2+2 \epsilon_{g}-\epsilon_{p_{1}}\right], \text { moreover } a=\tau_{m} b \text { and } \epsilon_{p_{1}} a=-\epsilon_{p_{0}} c .
\end{aligned}
$$

So, we can check, directly, that equation (3) is equivalent to equation

$$
\lambda^{2}-\frac{g\left(x_{0}\right)}{2}\left\{\left(2+2 \epsilon_{g}-\epsilon_{p_{1}}\right) \lambda+\left(-\epsilon_{p_{0}}\right)\left[\lambda e^{-\lambda \sigma}+\tau_{m} e^{-\lambda r}\right]-\epsilon_{p_{1}} \tau_{m}\right\}=0 .
$$




\section{Stability}

For a moment assume that in (8) either $2+2 \epsilon_{g}-\epsilon_{p_{1}}=0$ and $\epsilon_{p_{1}}<-\epsilon_{p_{0}}$ or $-\epsilon_{p_{0}} \tau_{m} g\left(x_{0}\right)>0$ and $\epsilon_{p_{1}} \tau_{m} g\left(x_{0}\right)<0$, so it is not true that all roots of the equation (9) have negative real part, once on the real axis $H(0)=\tau_{m}\left[\epsilon_{p_{1}}-\left(-\epsilon_{p_{0}}\right)\right]<0$ and $H(\lambda) \rightarrow \infty$ as $\lambda \rightarrow \infty$, so, there will be unbounded solutions of (1) and we do not uniform ultimate boundedness. As can been seen, from certain combination of the elasticities with time delay, erratic changes can arise around their fundamental values, which causes instability in the complex system of competition among the new technologies available.

Remark 2. From $0<17\left(-\epsilon_{p_{0}}\right)<2(3+\sqrt{22 \sqrt{2}}-\sqrt{2}) \epsilon_{p_{1}}$ it follows that

$$
\rho_{0}=:\left[\frac{32\left(\epsilon_{p_{1}}^{2}-\epsilon_{p_{0}}^{2}\right) c}{8 \epsilon_{p_{1}}^{2}-17 \epsilon_{p_{0}}^{2}}\right]^{\frac{1}{2}}<\left[\frac{8\left(\epsilon_{p_{1}}^{2}+\epsilon_{p_{0}}^{2}\right) c}{\epsilon_{p_{1}}^{2}(3-\sqrt{2})}\right]^{\frac{1}{2}}=: \rho_{1} .
$$

For a sake of simplicity, let be $\rho_{2}=: \frac{8 \sqrt{2} \epsilon_{p_{1}}^{\frac{3}{2}}}{(\sqrt{2}+6) \epsilon_{p_{1}}^{\frac{3}{2}}+2 \sqrt{\epsilon_{p_{1}}}+\sqrt{2\left(-\epsilon_{p_{0}}\right)}}$

Theorem 1. In addition to Assumption 1 and 2 we assume

$$
\begin{gathered}
\sqrt{g\left(x_{0}\right)}\left[2+2 \epsilon_{g}-\epsilon_{p_{1}}\right]<\min \left\{-\sqrt{2 g\left(x_{0}\right)}\left(-\epsilon_{p_{0}}\right) ;-2 \sqrt{\epsilon_{p_{1}} \tau_{m}}\right\} . \\
\text { If } 0<r<\sigma<\frac{\pi}{2}, 0<17\left(-\epsilon_{p_{0}}\right)<2(3+\sqrt{22 \sqrt{2}}-\sqrt{2}) \epsilon_{p_{1}} \text { and } \\
\rho_{0}<(-d)<\min \left\{\rho_{1}, \rho_{2}\right\}
\end{gathered}
$$

the equilibrium point $P_{0}$ is locally asymptotically stable ( $d$ is defined in (8)).

Proof. Using (7), (8) and since $0<-d<\rho_{2}$, we have $\bar{y}<2$ that is a condition of the Proposition 1. It follows from $\rho_{0}<(-d)<\rho_{1}$ that $4 a+(b+d)^{2}<4 c$ and $b<2 \sqrt{2 \Upsilon(a, c)\left(d^{2}-2 c\right)}$, where $\Upsilon(a, c)=\frac{c^{2}-a^{2}}{8 c^{2}+a^{2}}$. Since (11) holds, we can verify that the conditions of Theorem 1 and Proposition 1 of the Appendix are equivalent (see Remark 2). Then under conditions of Theorem (1), (9) all solutions has negative real part. It follows from Proposition 1 that the equilibrium point $P_{0}$ is locally asymptocally stable.

Theorem 1 gives sufficient conditions to ensure that the characteristic roots have negative real parts. This determines local stability of the equilibrium point for system (1). Under such conditions, markets agents have to take into account that dependence on memory in production of innovation is harmless, as for as the economic system operates close enough to the equilibrium point and indicators of technological innovation process are stable. We have demonstrated how market planners and technology planners must search for information in the memory of the system economic to improve the accuracy of them actions and make them efficient to choose reliable strategies in order to achieve them goals. 


\section{Fluctuations}

Nevertheless, once some parameters pass through critical values, Theorem 2 tells us that the system (1) loses stability and a Hopf Bifurcation will take place, that is, a family of periodic orbits bifurcates from the positive equilibrium point.

Theorem 2. In addition to Assumption 1 and 2 we assume that

$$
0<\sqrt{\left(-\epsilon_{p_{0}}\right) g\left(x_{0}\right)}<\tau_{m} \sqrt{\left(-\epsilon_{p_{0}}\right) g\left(x_{0}\right)}+\sqrt{\left[\left(-\epsilon_{p_{0}}\right) g\left(x_{0}\right) \tau_{m}^{2}+4 \tau_{m}\right]} .
$$

If $\sqrt{2}\left(-\epsilon_{p_{0}}\right)+\epsilon_{p_{1}}-2 \epsilon_{g}-2=0$, and $\sigma^{*}=\frac{\pi}{2}$, then there are $r^{*}$ and $y^{*}$ satisfying

$$
0 \leq\left(\frac{\pi}{2}-r^{*}\right) y^{*} \leq \frac{\pi}{2}
$$

so that $\left(\sigma^{*}, r^{*}, y^{*}\right)$ is a solution of the system (6). Moreover, if $\sin \left(\sigma^{*} y^{*}\right)>\left[4\left(\frac{y^{*}}{d}\right)^{2}+1\right]^{-1}$, $\Re\left(\left.\dot{\lambda}\left(\sigma^{*}, r^{*}, y^{*}\right)\right|_{x=0}\right) \neq 0$ and $\left(\sigma^{*}, r^{*}, y^{*}\right)$ is a Hopf bifurcation point and close to $P_{0}$, the system (1) oscilates (see (8)).

Proof. With a simple computation we verify that

$$
2 a b\left[\varrho(y)+y \varrho^{\prime}(y)\right]=4 y^{2}+2\left[d^{2}-\left(b^{2}-2 c\right)\right] y
$$

(see (6)). It follows from $\sqrt{2}\left(-\epsilon_{p_{0}}\right)+\epsilon_{p_{1}}-2 \epsilon_{g}-2=0$ that $d=-\sqrt{2} b$ (see the first item of the Proposition 1). Let be $m_{\varrho}=\frac{\epsilon_{p_{0}}^{2} g\left(x_{0}\right)-2 \tau_{m} \epsilon_{p_{1}}}{\tau_{1} \epsilon_{p_{0}}^{2} g\left(x_{0}\right)}$ and $n_{\varrho}=\frac{2 \tau_{m} \sqrt[4]{3 \epsilon_{p_{1}}^{5}\left(\epsilon_{p_{1}}^{2}-\epsilon_{p_{0}}^{2}\right)^{3}}}{3 \epsilon_{p_{0}}^{2}}$ and

$2\left(y^{*}\right)^{3}=\frac{n_{\varrho} \tau_{m}\left(\epsilon_{0} g\left(x_{0}\right)\right)^{2}}{4}$. It follows from (13) that $0 \leq m_{\varrho} \leq 1$ and $0 \leq n_{\varrho} \leq 1$. We can choose $0 \leq r^{*} \leq \sigma^{*}$ satisfying $0 \leq\left(\sigma^{*}-r^{*}\right) y^{*} \leq \sigma^{*}$ so that the straight line $z=m_{\varrho} y+n_{\varrho}$ is tangent to the graphic of the function $\varrho$ in (6) at the point $\left(y^{*}, \varrho\left(y^{*}\right)\right)$. Since $\varrho$ is a convex function on interval $(0, \infty)$, then $\varrho(y) \geq m_{\varrho} y+n_{\varrho}$ for all $y \geq 0$. Analogously, we choose $0 \leq \bar{r} \leq \sigma^{*}$ and $\bar{y}$ satisfying $0 \leq\left(\sigma^{*}-\bar{r}\right) \bar{y} \leq \sigma^{*}$ so that the straight line $z=m_{\sin } y+n_{\sin }$ is tangent to the graphic of the function sine defined in (6) at the point $\left(\bar{y}, \sin \left(\sigma^{*}-\bar{r}\right) \bar{y}\right)$. It is easy see that

$$
m_{\text {sin }}=m_{\sin }(\bar{r}, \bar{y})=\left(\sigma^{*}-\bar{r}\right) \cos \left(\sigma^{*}-\bar{r}\right) \bar{y}
$$

and

$$
n_{\text {sin }}=n_{\text {sin }}(\bar{r}, \bar{y})=\sin \left(\sigma^{*}-\bar{r}\right) \bar{y}-\left(\sigma^{*}-\bar{r}\right) \bar{y} \cos \left(\sigma^{*}-\bar{r}\right) \bar{y} .
$$

Since $r^{*}$ and $y^{*}$ were chosen so that $\varrho^{\prime}\left(y^{*}\right)=m_{\varrho}$ and $\varrho\left(y^{*}\right)=n_{\varrho}$, it follows from (5) and Implicit Function Theorem that the unique solution of the system

$$
\left\{\begin{array}{l}
m_{\sin }(\bar{r}, \bar{y})=m_{\varrho} \\
n_{\sin }(\bar{r}, \bar{y})=n_{\varrho}
\end{array}\right.
$$

will be $\left(r^{*}, y^{*}\right)$. 
By using implicit derivative in (3) we can show that

$$
\Re\left(\left.\dot{\lambda}(\sigma, r, y)\right|_{x=0}\right)=b y^{2}[2 y \sin \sigma y-d \cos \sigma y]+r b a \cos (\sigma-r) y .
$$

Because $\sin \left(\sigma^{*} y^{*}\right)>\left[4\left(\frac{y^{*}}{d}\right)^{2}+1\right]^{-1}$, we have $\Re\left(\left.\dot{\lambda}\left(\sigma^{*}, r^{*}, y^{*}\right)\right|_{x=0}\right)>0(d$ is defined in (8)).

Remark 3. If the elasticities of the functions involved in model (1) satisfy the conditions of Theorem 2 we are able to localize a non-null purely imaginary root of equation (9) and to show that as the delay $\sigma$ crosses the crtical value $\sigma^{*}$ there are two simple roots of equation (9) crossing transversely the imaginary axis from left to right, while all others have negative real part. We can also verify that $\left(\sigma^{*}, r^{*}\right)$ determines a sequence $\left\{\left(\sigma_{p}^{*}, r_{q}^{*}\right)\right\}_{(p, q) \in N^{2}}$ of critical values that lie in a smooth manifold $\mathscr{K}$ defined by system (14). Using Hopf-bifurcation theory we show that the manifold $\mathscr{K}$ can be chosen in such way that each one of this critical values, near the equilibrium point $P_{0}$, is associated to a nonconstant periodic solution of system (1).

\section{Conclusions}

With endogenous framework, the stabilization of competition on a market that operates with several manufacturers using same technologies, is accurately analyzed. It is clear from Theorems 1 and 2 that production delays in commodities markets are potentially destabilizing factors. Theorem 1 shows that planners can search for information in the memory of an economic system without doing harm to parameter systems governing technological innovation process. Theorem 2 shows that if parameters are close to the boundary of the stability region the system undergoes Hopf bifurcation. With this phenomenon the technological innovation process becomes unstable and fluctuating. This lead us to believe that our analysis of the model (1) is able to offer technology planners a reasonable explanation for cyclical behaviour in competitive markets, and it suggests how market agents must act to avoided fluctuations.

\section{References}

[1] G. Barbirol and D. Ritelli. Dymamical systems in analysing competitiveness and coexistence among technologies. International Journal of Systems Science, 28(4):347-356, 1997.

[2] J. Bléair and M. C. Mackey. Consumer memory and price fluctuations in commodity markets: an integrodifferential model. Journal of Dynamic of Differential Equations, 1(3):299-325, 1989.

[3] H. L. Freedman. Stability analysis of a prey-predator system with mutual interference and density-dependent death rates. Bulletin of Mathematical Biology, 41(1):67-78, 1979.

[4] J. K. Hale and S. M. V. Lunel. Introduction to Functional Differential Equations. Springer Verlag - New York - 1993, 1993. 
[5] T. Howroyd and A. Russel. Cournot oligopoly models with time delays. Journal of Mathematical Economics, 1(13):97-103, 1981.

[6] C. Huang, C. Peng C., Chen, and F. Wen. Dynamics analysis of a class of delayed economic model. Abstract and Applied Analysis, 2013(Article ID 962738):12 pages, 2013.

[7] W. Huang. Information lag and dynamic stability. Journal of Mathematical Economics, 44(1):513-529, 2013.

[8] C. M. Mackey. Commodity price fluctuation: Price dependent delays and nonlinearities explanatory factors. 48(1):497-509, 1989.

[9] A. Matsumoto and F. Szidarovszky. Nonlinear delay monopoly with bounded rationality. Chaos Solitons and Farctais, 45(4):507-519, 2012.

[10] D. Ritelli, G. Barbirol, and P. Fabbri. Predation among technologies on market: A modelistic analysis. Journal of Mathematical Economics, 27(1):347-374, 1997.

[11] Y. Takeuchi and T. Yamamura. Analysis of the kaldor model with time delay: monetary policy and government budget constraint. Nolinear Analysis Real World Applications, 5(1):277-308, 2004.

[12] E. Wolfstetter. Fiscal policy and the classical growth cycle. Journal of Economics, 42(4):375-393, 1982.

[13] T. Yamamura. Analysis of the Kaldor Model with Time Delay: Monetary Policy and Government Budget Constraint. PhD thesis, Graduate School of Science and Engineering Shizuoka University, 2001.

[14] K. Zhu and J. P. Weyant. Strategic decisions of new technology adoption under asymmetric information: a game-theoretic model. Decision Sciences, 34(4):643-675, 2003.

\section{Appendix}

In order to simplify notation we set

$$
\bar{x}=\frac{b+\sqrt{b^{2}+2 a}}{2}, \bar{y}=\frac{\bar{x}}{2}+\frac{b}{2}-\frac{d}{2}+\sqrt{\left(\frac{\bar{x}}{2}+\frac{b}{2}-\frac{d}{2}\right)^{2}+a+c .}
$$

Unfortunately, the analysis is not easy, since it involves hard computations.

Proposition 1. Assume (7) are satisfied and

(i) $d<\min \{-\sqrt{2} b,-\sqrt{2 c}\}, 2 r<2 \sigma<\pi$,

(ii) $4 a+(b+d)^{2}<4 c, \bar{y}<2$, 
(iii) $b<2 \sqrt{2 \Upsilon(a, c)\left(d^{2}-2 c\right)}$, where $\Upsilon(a, c)=\frac{c^{2}-a^{2}}{8 c^{2}+a^{2}}$.

Then, there is $\epsilon_{0}>0$ so that if $\bar{x}<\epsilon_{0}$, all roots of the equation (3) have negative real part.

Proof. We observe that $a, b$ and $c$ are positive, $d$ is negative and $c>a$. We suppose that $y=0$ in (4). The system reduces to

$$
x^{2}-\left(b e^{-\sigma x}+d\right) x-a e^{-r x}+c=0
$$

If $\vartheta(x)=x^{2}-\left(b e^{-\sigma x}+d\right) x-a e^{-r x}+c$ then $\vartheta(0)>0$. If $x>0$, the first inequality in (i) gives $\vartheta(x)>x^{2}-(b+d) x-a+c>0$. From this it follows that $p(\lambda)=0$ has no positive solution with null imaginary part (see (3)).

Let consider the sets $S_{i}$ for $i \in\{0,1,2, \cdots, 6\}$ given by

$$
\begin{aligned}
& S_{0}=\{(x, y) \in R: 0<x \leq \bar{x} \text { and } 0<\bar{y} \leq y\} ; \\
& S_{1}=\left\{(x, y) \in R^{2}: 0<4 \sigma y \leq \pi, \text { and } 2 x>b\right\} ; \\
& S_{2}=\left\{(x, y) \in R^{2}: \pi \leq 4 \sigma y \leq 2 \pi \text { and } 0<y \leq x\right\} ; \\
& S_{3}=\left\{(x, y) \in R^{2}: \pi \leq 2 \sigma y \leq 2 \pi \text { and } x>0\right\} ; \\
& S_{4}=\left\{(x, y) \in R^{2}: 0<y \leq x \text { and } y \geq \bar{x}\right\} ; \\
& S_{5}=\left\{(x, y) \in R^{2}: 0<x \leq y \text { and } x \geq \bar{x}\right\} ; \\
& S_{6}=\left\{(x, y) \in R^{2}: \pi \leq 4 \sigma y \leq 2 \pi \text { and } 0<x \leq y\right\} .
\end{aligned}
$$

For each $(x, y) \in R_{+}^{2}$, the first equation in (4) becomes equivalent to

$$
\Gamma_{1}(x, y)=0
$$

where

$$
\Gamma_{1}(x, y)=x-y-\frac{d x-c}{x+y}-b e^{-x \sigma} \zeta_{1}(x, y)-\frac{e^{-x r}}{x+y} a \cos y r
$$

and

$$
\zeta_{1}(x, y)=(x+y)^{-1}[x \cos y \sigma+y \sin y \sigma]
$$

We note that

$$
\left|\zeta_{1}(x, y)\right| \leq 1 \text { and } \frac{-a e^{-r x}}{x+y} \leq \frac{-a e^{-r x} \cos r y}{x+y} \leq \frac{a e^{-r x}}{x+y} \leq \frac{a e^{-r x}}{y} .
$$

If $\epsilon^{*}(x, y)=-y^{2}+(b+x-d) y+a+c$, then $y \Gamma_{1}(x, y)<\epsilon^{*}(x, y)$ since $-d x<-d(x+y)$. If $(x, y) \in S_{0}$, we can verify that $\epsilon^{*}(x, y)<0$ and so, equation $\mathrm{A} 3$ has no solution belonging to $S_{0}$. In fact, the function $y(x)=\frac{(b+x-d)+\sqrt{(b+x-d)^{2}+4(a+c)}}{2}$ is increasing in $x$ and gives a positive solution for the second degree equation in $y$ given by $\epsilon^{*}(x, y)=0$.

The second equation in (4) is equivalent to

$$
\Gamma_{2}(x, y)=2 x y-d y+b e^{-\sigma x}[x \sin \sigma y-y \cos \sigma y]+a e^{-r x} \sin r y=0 .
$$


Since $\Gamma_{2}(x, y)>y(2 x-b)$ e $2 x>b$, there is no solution of equation A5 for $(x, y)$ belongs to $S_{1}$.

It is easy to see that $x \sin \sigma y-y \cos \sigma y \geq 0$ for $(x, y) \in S_{2}$. Moreover, for all $(x, y) \in S_{2}$, we have $\Gamma_{2}(x, y) \geq 2 x y-d y+a e^{-r x} \sin r y>0$. Thus, there is no solution of (A3) that belongs to $S_{2}$.

Let consider $(x, y) \in S_{3}$, then $\Gamma_{2}(x, y) \geq 2 x y-d y$ which is positive because $d<0$. Hence, there is no solution of (A3) that belongs to $S_{3}$.

For each $(x, y) \in S_{4}$, we define $\zeta_{2}(x, y)=(x y)^{-1}[x \sin y \sigma-y \cos y \sigma]$. we can check directly that $y \zeta_{2}(x, y) \geq-2$ and $y \sin y r \geq-x$. Then, $\Gamma_{2}(x, y)>\left(x^{-1} y^{-3}\right)\left[2 y^{2}-2 b y-a\right]$, which is positive if $y \geq \bar{x}$ (see A1), whence (A5) has no solution at $S_{4}$.

Let $(x, y) \in S_{5}$. Because $x \zeta_{2}(x, y) \geq-2$ and $(x y)^{-1} \sin (y r) \geq-x^{-2}$, then $\Gamma_{2}(x, y)>\left(x^{-3} y^{-1}\right)\left[2 x^{2}-2 b x-a\right]$ that is positive if $x \geq \bar{x}$ (see (A1)) and so, has no solution into $S_{5}$.

We assume $(x, y) \in S_{6}$, since $x y \geq x^{2}$ and $-b y \frac{\sqrt{2}}{2}<-b y e^{-\sigma x} \cos \sigma y$, $\sigma \Gamma_{2}(x, y)>2 x^{2} \sigma-\frac{\pi}{4}(d+b \sqrt{2})$ is positive if $d<-b \sqrt{2}$ (see (1)). Hence, there is no solution of (A3) that belongs to $S_{6}$.

Finally, the system (4) has no purely imaginary solution. If the roots of the system (4) with null real part exist, they must be roots of the system (5)

We suppose $y \neq 0$ is a solution of (5), then we must have $\sin (\sigma y-r y)=\varrho(y)$ (see (6))). It is clear that $\varrho(y)=-\varrho(-y)$, thus we will consider only $y>0$. A direct calculation shows that $2 b a y^{2} \dot{\varrho}(y)=3 y^{4}+\left(d^{2}-b^{2}-2 c\right) y^{2}-\left(c^{2}-a^{2}\right)$ and that

$$
y_{0}=\left[\frac{\sqrt{\left[d^{2}-\left(2 c+b^{2}\right)\right]^{2}+12\left(c^{2}-a^{2}\right)}-\left[d^{2}-\left(2 c+b^{2}\right)\right]}{6}\right]^{\frac{1}{2}},
$$

is a global minimum point of $\varrho$ on the interval $(0, \infty)$. Since

$$
a b y^{2} \dot{\varrho}(y)=a b y \varrho(y)+y^{4}-\left(c^{2}-a^{2}\right),
$$

then $y_{0}^{4}=\left(-d^{2} y_{0}^{2}+b^{2} y_{0}^{2}+2 c y_{0}^{2}-a^{2}+c^{2}\right) / 3$ and $3 a b y_{0} \varrho\left(y_{0}\right)=2\left(c^{2}-a^{2}\right)+\left(d^{2}-2 c-b^{2}\right) y_{0}^{2}$, the inequality of (3) and the first inequality in (1) implies that $\varrho\left(y_{0}\right)>1$. Therefore, (3) has no purely real root on the right half complex plane. By general arguments on the compacity of the interval $\left[0, \frac{\pi}{4}\right]$ and the continuity of $\varrho$, we arrive at the existence of a $\epsilon_{0}>0$ so that System (4) has no solution belonging to $S_{\epsilon_{0}}=\left\{(x, y) \in R^{2}: 0 \leq x<\epsilon_{0}, \quad 0 \leq 4 \sigma y \leq \pi\right\}$. If $R_{+}^{2}=\left\{(x, y) \in R^{2}, x>0, y>0\right\}$, the conditions (i), (ii), (iii) and $\bar{x}<\epsilon_{0}$ imply that $R_{+}^{2} \subset \mathscr{S} \cup S_{\epsilon_{0}}$, where $\mathscr{S}=\bigcup_{i=0}^{6} S_{i}$ (see (A2)). So, it can proven that System (4) has no solution that lies in $\operatorname{Cl}\left(R_{+}^{2}\right)$ and the proof of the Theorem is complete. 